\title{
THE EFFICACY OF SQUAMOUS CELL CARCINOMA ANTIGEN IN THE DIFFERENTIAL DIAGNOSIS OF ORAL SQUAMOUS CELL CARCINOMA
}

\author{
Maha Abdelkawy*, Shereen Ali **, Olfat shaker ${ }^{* * *}$ and Ahmed Nabil Fahmi****
}

\begin{abstract}
Background and Objectives: Oral squamous cell carcinoma (OSCC) is a major oral cancer representative. Squamous cell carcinoma antigen (Scc-AG) is detected in squamous cell carcinoma of oral tissues and other different tissues. It has been used as a biomarker for OSCC and hence the aim of this study was to detect if Scc-Ag can differentiate between malignant lesions and suspicious chronic inflammatory lesions..
\end{abstract}

Methods: Forty-five participants divided into OSCC group and two control groups: oral lichen planus as an inflammatory disease (positive control) and healthy volunteers (negative control). Clinical staging and grading of OSCC was recorded. A double-antibody sandwich enzyme-linked immunosorbent assay was used to evaluate the level of Scc-AG in serum and saliva.

Results: Statistically significant difference for serum and salivary Scc-Ag, was found between the groups; OSCC, OLP on one hand and healthy controls on the other hand. No statistically significant difference was found between OSCC and OLP control groups in serum levels, while for salivary Scc-Ag a statistically significant difference was found between these two groups, with the higher values in OLP. Staging and grading in OSCC group were not found to be related to Scc-Ag level.

Conclusion: Serum Scc-Ag cannot be used as a biomarker to differentiate between oral malignant and chronic inflammatory lesions. Salivary Scc-Ag is more reliable than serum Scc-Ag in differential diagnosis of OSCC.

KEYWORDS: Squamous Cell Carcinoma Antigen, Biomarker, Oral squamous cell carcinoma, Oral lichen Planus, Salivary biomarkers.

\footnotetext{
* Department of Oral Medicine, and Periodontology, Faculty of Dentistry, Beni-Suef University, Beni-Suef, Egypt. ** Department of Oral Medicine and Periodontology, Faculty of Oral and Dental Medicine, Cairo University. *** Department of Biochemistry, Faculty of Medicine, Cairo University. **** Department of Oral Pathology, Faculty of Dentistry, Beni-Suef University, Beni-Suef, Egypt.
} 


\section{INTRODUCTION}

Oral squamous cell carcinoma (OSCC) is a malignant tumor developed from stratified squamous epithelium of the oral mucosa representing more than $90 \%$ of oral cancer with destructive and invasive behavior. It is considered to be curable if early detected, yet the majority of cases are lately discovered which subsequently leads to poor prognosis. It is mainly found in old age especially with certain risk factors. (Rivera and Venegas, 2014, Tampa et al., 2018).

Inflammation has been postulated to play a role in the carcinogenesis process of the disease. It is now believed that interaction with inflammatory cells can be a promoting factor in tumor growth and propagation or a hindering factor depending on the inflammatory mediators secreted by the aggregated inflammatory cells in the tumor vicinity (Laliberté, 2019).

Squamous cell carcinoma Antigen (Scc-Ag) is a tumor associated antigen of squamous cell carcinoma that was first discovered in uterine cervix. It is from the serine protease inhibitor family with two different forms (Scc-Ag1, Scc-Ag2) encoded by two different genes on the same chromosome (Kato and Torigoe, 1977).

Scc-Ag is present in the spinous and granular layers of normal mucosa but unexpressed except in non-physiological conditions where it is secreted in tissues and in biological fluids. Proinflammatory cytokines stimulate Scc-Ag expression. Hence it is said that it aids in the restoring homeostasis through proteolysis control in inflamed epithelial cell (Cataltepe et al., 2000, Hayat, 2005).

Some oral lesions as oral lichen planus (OLP), which is a cell mediated inflammatory condition characterized by chronicity, might present as a suspicious clinical entity, and raises the question of malignant transformation. (Alrashdan et al, 2016).

This led to the importance of pursuing search for biomarkers for early detection of oral malignant transformation as well as for diagnostic and prognostic value in oral cancer. This mainly includes serum biomarkers and recently salivary biomarkers have been studied (Radhika et al., 2016, Tampa et al., 2018).

Accordingly, our research question has been formulated, if Scc-Ag as a biomarker can differentiate between inflammatory lesions and those with malignant transformation.

\section{SUBJECTS AND METHODS}

This cross-sectional case control study was permitted by the Research Ethics Committee with ID number \#FDBSUREC/21012020/AM and was performed in full accordance with the World Medical Association Declaration of Helsinki 1975, revised in 2003. All patients had been given the suitable treatment after their contribution in the study.

Forty-five participants were included in this study. They were divided in to three groups. (figure 1)

(I) First control group (negative control): including Fifteen healthy volunteers

(II) Second control group (positive control): Fifteen symptomatic OLP patients with histopathological diagnosed bullous or erosive/ ulcerative

(III) OSCC group: including fifteen patients with histopathological diagnosed OSCC lesions. Clinical staging and grading were done according to the guidelines of the 2006 American Joint Committee on Cancer (tumor-node-metastasis classification) (Greene et al., 2010)

Exclusion Criteria: Exclusion was done according to the following: (1) No lactating or pregnant females; (2) None of the participants had a systemic condition or was under medications; (3)No other mucosal lesions detected; and (4) Avoiding inflammation in the periodontium. The participants ages ranged from thirty-two to sixty-five. 


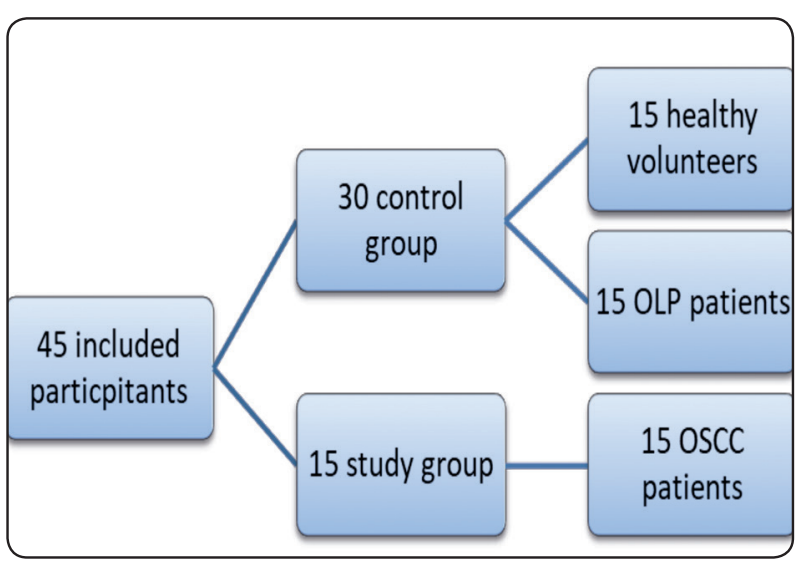

Fig. (1) Flow chart for participants in the study

\section{Collection of salivary samples}

According to Navazesh,1993 standard technique, the samples of Whole unstimulated saliva were collected from different groups participants. All Participants were asked to inhibit drinking and eating for 30 minutes. Then they were asked to swallow and bend their hear head forward to expectorate saliva in a previously given tube. This continued for 5 minutes. The samples were then preserved at $-20^{\circ} \mathrm{C}$ for later assessment

\section{Blood sample collection:}

Three milliliter blood was collected from each subject in ethylenediaminetetraacetic acid (EDTA) tubes by venipuncture at the antecubital fossa. The tubes were maintained in ice and transported to the biochemistry lab at Faculty of Medicine, Cairo University, where it was stored at $-70^{\circ} \mathrm{C}$ until all samples were collected. All collected samples were given specific serial number (patient's information was not shown on the samples labels).

\section{Evaluation of Salivary and serum squamous cell carcinoma related antigen}

Evaluation of Salivary and serum Scc-Ag was done by enzyme-linked immunosorbent assay (ELISA). The ELISA unit was given by Bioassay Innovation Research facility; Shanghai, China (Cat. No-E2108Hu). The level of Human squamous cell carcinoma antigen (SCC-AG) in samples was assessed by using a double-antibody sandwich ELISA. Add SCC-AG to monoclonal antibody Enzyme well which is pre-coated with SCC-AG monoclonal antibody, incubation; then, add SCCAG antibodies labeled with biotin, and combined with Streptavidin-HRP to form immune complex; then carry out incubation and washing again to remove the uncombined enzyme. Then add Chromogen Solution A, B, the color of the liquid changes into the blue, and at the effect of acid, the color finally becomes yellow. The color chroma and the Human Substance SCC-AG concentration of sample were positively correlated to each well to stop the reaction (immediate change from blue to yellow)

\section{Statistical Analysis}

The mean and standard deviation values were calculated for each group in each test. Data were explored for normality using KolmogorovSmirnov and Shapiro-Wilk tests, data showed parametric (normal) distribution except for grades, clinical stages, pain and ulcer scores showed nonparametric (not normal) distribution.One-way ANOVA followed by Tukey-post hoc test was used to compare between more than two groups in non-related samples for parametric data. Pearson and Spearman correlation tests was used to find the correlation between different variables in parametric and non-parametric data, respectively. The significance level was set at $\mathrm{P} \leq 0.05$. Statistical analysis was performed with IBM ${ }^{\circledR} \quad$ SPSS ${ }^{\circledR}$ Statistics Version 20 for Windows.

\section{RESULTS}

\section{I) Base line characteristics}

No statistically significant difference was found among gender distributions in the three groups. As for age, no statistically significant difference was found between first and second control groups; both showed statistically significantly lower mean age values than OSCC (Age Mean=38.4,42.4,51.7 respectively). 
II) Relation between different groups in each marker Scc-Ag (ng/ml) serum and saliva:

For both serum and salivary Scc-Ag, a statistically significant difference was between the groups; OSCC, OLP control and healthy control; where $p<0.001$. A statistically significant difference was found between healthy control and each of OSCC and OLP control group where $\mathrm{p}<0.001$ for both salivary and serum Scc-Ag. As for the difference between OSCC group and OLP control group, there was no statistically significant difference in $\operatorname{serum}(\mathrm{p}=0.431)$ while for salivary Scc-Ag, a statistically significant difference was found $(\mathrm{p}<0.001)$

The highest mean value was found in OLP control group followed by OSCC, while the lowest mean value was found in healthy Control group for SCC-Ag in serum and saliva.. (Table 1 and Figure 2)

\section{III) Correlation between different groups:}

There was non-significant weak positive relationship between Scc-Ag serum and Scc-Ag saliva, in each group independently; OSCC $[\mathrm{r}=$ $0.060, \mathrm{p}$ (2-tailed) $=0.840]$, OLP control group $[\mathrm{r}$ $=0.160, \mathrm{p}(2$-tailed $)=0.570]$ and control healthy group $[\mathrm{r}=0.160, \mathrm{p}(2$-tailed $)=0.570]]$ (Figure 3$)$.

The receiver operating characteristic (ROC) curve analysis of the two markers for differentiation between OSCC and OLP showed diagnostic accuracy of Scc-Ag in saliva $=79.3 \%$ at cut-off value $=6.01 \mathrm{ng} / \mathrm{ml}$ and accuracy of Scc- $\mathrm{Ag}$ in serum $=65.5 \%$ at cut-off value $=3.61 \mathrm{ng} / \mathrm{ml}$, with no statistical significance.

TABLE (1): The mean, standard deviation (SD) of markers in different groups.

\begin{tabular}{|c|c|c|c|c|}
\hline \multirow{2}{*}{ Variables } & \multicolumn{4}{|c|}{ Markers } \\
\cline { 2 - 5 } & Scc-Ag (ng/ml) serum & Scc-Ag (ng/ml) saliva \\
\cline { 2 - 5 } & Mean & SD & Mean & SD \\
\hline Carcinoma & $3.59^{\mathrm{a}}$ & 0.53 & $5.21^{\mathrm{b}}$ & 1.02 \\
\hline OLP & $4.00^{\mathrm{a}}$ & 1.39 & $6.19^{\mathrm{a}}$ & 0.68 \\
\hline Control & $0.92^{\mathrm{b}}$ & 0.26 & $2.09^{\mathrm{c}}$ & 0.40 \\
\hline p-value & \multicolumn{3}{|c|}{$<\mathbf{0 . 0 0 1}^{*}$} & \multicolumn{4}{|c|}{$\mathbf{0 . 0 0 1}^{*}$} \\
\hline
\end{tabular}

Means with different small letters in the same column indicate statistically significance difference *; significant $(p<0.05) \quad n s ;$ non-significant $(p>0.05)$

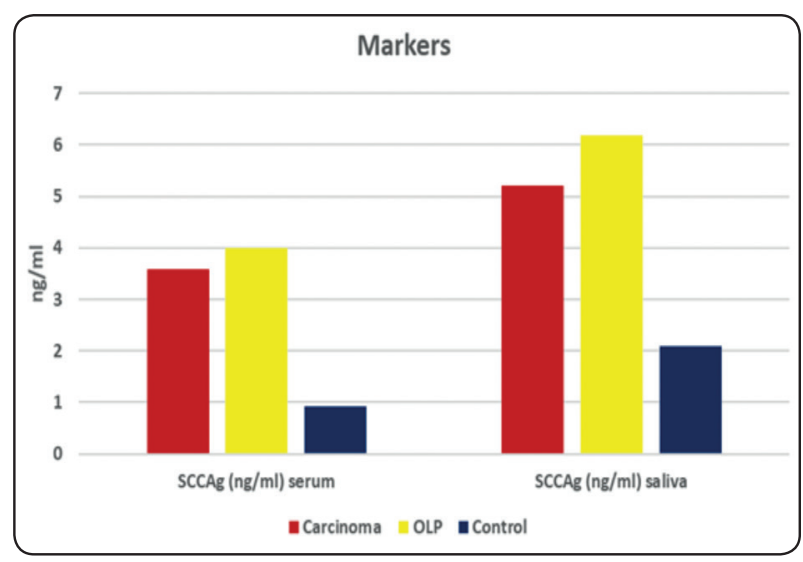

Fig. (2): Bar chart representing markers for different groups.

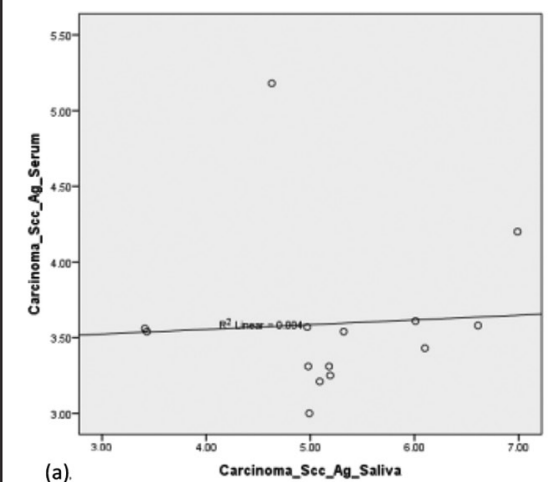

(a)

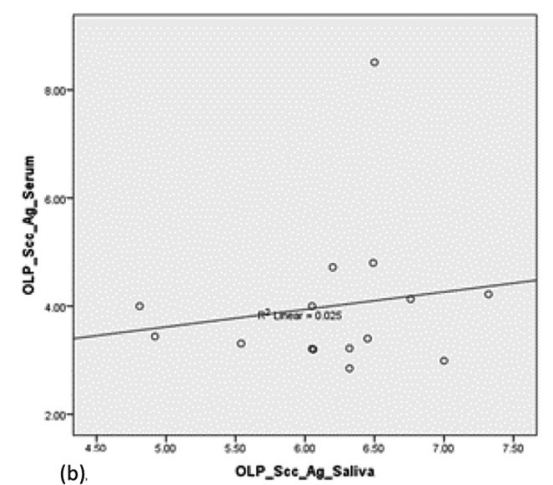

(b)

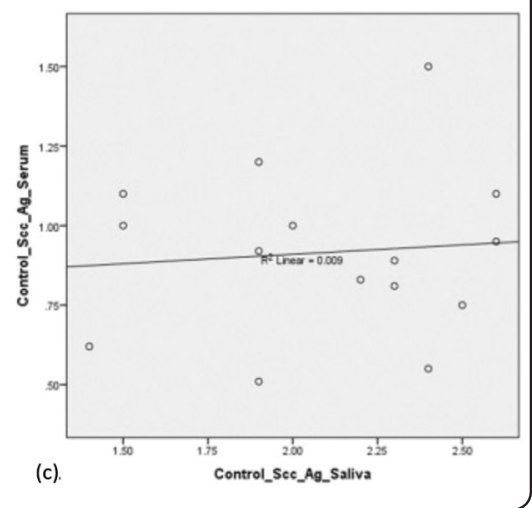

Fig. (3): correlation between serum Scc-Ag and salivary Scc-Ag (a) in OSCC group, (b), in OLP control group, (c)in healthy control group. 
IV) Correlation between grades and Clinicals stages of OSCC group and Scc-Ag:

There was non-significant relationship between serum and salivary Scc-Ag and the grades and clinical stages of OSCC group.

TABLE (2): Correlation between grades and Clinicals stages of OSCC group and serum and salivary Scc-Ag

\begin{tabular}{|c|c|c|}
\hline Variables & \multicolumn{2}{|l|}{ Carcinoma } \\
\hline \multirow{2}{*}{ Scc-Ag serum and grades } & Correlation coefficient & -0.352 \\
\hline & Sig. (2-tailed) & 0.217 \\
\hline \multirow{2}{*}{$\begin{array}{c}\text { Scc-Ag serum and clinical } \\
\text { stage }\end{array}$} & Correlation coefficient & -0.417 \\
\hline & Sig. (2-tailed) & 0.138 \\
\hline \multirow{2}{*}{ Scc-Ag saliva and grades } & Correlation coefficient & 0.166 \\
\hline & Sig. (2-tailed) & 0.570 \\
\hline \multirow{2}{*}{$\begin{array}{c}\text { Scc-Ag saliva and clinical } \\
\text { stage }\end{array}$} & Correlation coefficient & 0.311 \\
\hline & Sig. (2-tailed) & 0.279 \\
\hline
\end{tabular}

\section{DISCUSSION}

OSCC represents about $90 \%$ of all oral cancer and is characterized by its local invasion ability and its critical metastatic activity. Certainly, its early diagnosis will aid in having better prognosis (Omura, 2014; Tampa et al., 2018). Scc-Ag originates from squamous cell carcinoma associated antigen. It has been widely used as serological marker for squamous cell carcinomas in different tissues (Fu et al. 2019, Guo et al.2020) OLP is a cell mediated chronic inflammatory disease with only $1.1 \%$ of patients developing OSCC (Aghbari et al., 2017).

The aim of the current study was to identify if Scc-Ag can be useful as a biomarker for the differentiation between oral inflammatory lesions and conditions with malignant transformation. We examined forty-five participants: OSSC patients, OLP patients representing inflammatory diseases control group and healthy control volunteers. Scc$\mathrm{Ag}$ was detected in serum and saliva to compare between their results and to ascertain the ability of using salivary biomarker as being easier and more attainable.

Our demographic data showed no statistically significant difference in gender distribution among the three groups. Regarding age, there was no statistically significant difference found between OLP and healthy control groups. Both showed statistically significantly lower mean age values than OSCC. This is in accordance with OSCC criteria where its majority occurs in older age group patients (Rivera and Venegas, 2014)

The results of the current study showed that OLP control group had the highest mean value of Scc$\mathrm{Ag}$ in serum and saliva. For serum Scc-Ag, there was statistically significant difference between the healthy control group and, OLP control group, OSCC, showing that OSCC is nearly as OLP with no statistical significance difference between them. On the other hand, for salivary Scc-Ag there was statistically significant difference among the 3 groups independently. This implies that Scc-Ag is an inflammatory biomarker rather than malignant transformation indicator.

As cellular mediated immunity plays a main role in the pathogenesis of OLP with T-helper cell playing a pivot role. Numerous inflammatory cytokines are produced consequently and are held to be partially responsible for the characteristic features and chronicity of OLP (Alrashdan et al, 2016). Pro-inflammatory cytokines are abundant in OLP, therefore explaining the high levels of Scc-Ag in OLP that even exceed OSCC in case of Saliva.

In accordance to our results A previous study stated that SCC-Ag was found to be elevated in OLP and was used to monitor the treatment efficacy as well as its direct positive relation with clinical remission and exacerbation of the disease (Sun and Chiang, 2001) 
Furthermore, Watanabe et al., studied the level of Scc-Ag in psoriasis which is a well-known chronic inflammatory disease with no malignant transformation and found serum Scc-Ag to be positively related with severity and efficacy of treatment applied (Watanabe et al., 2016)

Our results showed that salivary Scc-Ag in OLP control group was statistically significantly higher than OSCC group. This could be explained by that OLP is mainly a chronic inflammatory disease with proinflammatory cytokines playing the main role as previously mentioned (Alrashdan et al, 2016).On the other hand inflammation partially plays a role in OSCC where its presence is positively corelated to its severity (Tampa et al., 2018).

Moreover, Scc-Ag is normally present in all biological fluids due to passive secretion during cells desquamation. Given that it's expression is stimulated by proinflammatory cytokines, SccAg presence in saliva, one of the vital body fluids, increases in case of chronic inflammatory diseases as OLP, especially that it is in direct contact with the lesion (Hayat, 2005).

Since inflammation has a role in carcinogenesis, numerous markers of inflammation has been studied in OSCC as prognostic markers $(\mathrm{C}$ reactive protein, nuclear factor kappa beta, cytokines, IL-6, IL-8 Cyclooxygenase and matrix metalloproteinases), yet, no reliable biomarker for early detection of malignant transformation was found (Pellicioli et al., 2017, Tampa et al., 2018).

Our results showed non-significant weak positive relationship between serum and salivary Scc-Ag in each study group independently. This implies that salivary Scc-Ag could be reliable as much as serum Scc-Ag. This is in accordance with Hedge et al who reviewed different salivary biomarkers for oral cancer and stated that salivary approach is superior to serum regarding its effectiveness, noninvasiveness, safety, and patient compliance. (Hegde et al., 2018). On the contrary, some demerits of salivary biomarkers have been mentioned in literature as lack of collection of samples standardization, processing, and storage (Radhika et al., 2016)

However, the salivary and serum Scc-Ag were not found to be related to cancer staging and grading in OSCC group, which was controversial with another study detecting the clinical significance of preoperative Scc-Ag on OSCC. Serum Scc-Ag level above $2 \mathrm{ng} / \mathrm{mL}$ was related to higher risk for skin and bone invasion as well as lymph node metastasis (Lin et al., 2011).

A recent study reported that preoperative level of serum Scc-Ag in cervical squamous cell carcinoma is related to distant recurrence and is considered an independent factor for recurrence and survival (Guo et al., 2020). Our conflicting results may be due to the small sample size. Another study researched serum Scc-Ag in hepatocellular carcinoma stated that there was no difference between Scc-Ag level among hepatocellular carcinoma, liver cirrhosis and healthy control (El-Bassuoniaa et al., 2020)

$\mathrm{Scc}-\mathrm{Ag}$ is a substantiated biomarker that has been found in normal tissues containing squamous cells, with increased expression in cases of chronic inflammation. Its level is directly related to the amount of proinflammatory cytokines and so found more in chronic inflammatory disease and therefore may be used for monitoring disease activity and following up treatment efficacy in inflammatory disease (Watanabe et al., 2016). Its effect in differentiating between malignant and inflammatory conditions is still unproved. Salivary Scc-Ag is a promising biomarker showing results as reliable or even more than serum Scc-Ag and could be used in monitoring inflammatory disease activity and follow up efficiency of treatment.

Funding: self-funded

Conflict of interest: All authors declare no conflict of interest 


\section{REFERENCES}

- Aghbari SMH, Abushouk AI, Attia A, et al. Malignant transformation of oral lichen planus and oral lichenoid lesions: A meta-analysis of 20095 patient data. Oral Oncol. 2017;68:92-102. doi: 10.1016/j.oraloncology.2017.03.012

- Alrashdan M, Cirillo N and McCullough M. Oral lichen planus: a literature review and update. Arch Dermatol Res 2016 308:539-551.

- Cataltepe S, Gornstein ER, Schick C, et al. Co-expression of the squamous cell carcinoma antigens 1 and 2 in normal adult human tissues and squamous cell carcinomas. J Histochem Cytochem. 2000;48(1):113-122. doi: $10.1177 / 002215540004800112$

- $\quad$ El-Bassuoniaa MA, El-Edela RH, Eman A. Gawesha EA and Mandoura SS. The diagnostic value of serum squamous cell carcinoma antigen for prediction of hepatocellular carcinoma. http://www.mmj.eg.net on Wednesday, February 26, 2020, IP: 41.68.254.172.

- $\quad$ Fu J, Wang W, Wang Y, Liu C and Wang P. The role of squamous cell carcinoma antigen ( $\mathrm{SCC} \mathrm{Ag)} \mathrm{in} \mathrm{outcome}$ prediction after concurrent chemoradiotherapy and treatment decisions for patients with cervical cancer. Radiation Oncology (2019) 14:146 https://doi.org/10.1186/s13014019-1355-4

- Greene FL, Page DL, Fleming ID, et al. AJCC Cancer Staging Manual, 6th ed. New York, NY: Springer; 2010.

- Guo Q, Zhu J, Wu Y, et al. Predictive value of preoperative serum squamous cell carcinoma antigen (SCC-Ag) level on tumor recurrence in cervical squamous cell carcinoma patients treated with radical surgery: A single-institution study. Eur J Surg Oncol. 2020;46(1):131-138. doi: 10.1016/j.ejso.2019.08.021.

- Hayat M., in Handbook of Immunohistochemistry and in Situ Hybridization of Human Carcinomas, London, Science direct,2005, vol. 3 .

- Hegde ND, Hegde MN, Priya G An Insight on salivary biomarkers for oral Cancer. J Dent Craniofac Res. ,2018, mVol.3 No.2:8.

- Kato H and Torigoe T. Radioimmunoassay for tumor antigen of human cervical squamous cell carcinoma. Cancer 1977; 40:1621-1628.1977.
- Laliberté C. (2019) A Pilot Study of the Inflammatory Response in Oral Squamous Cell Carcinoma by Saliva Analysis. A thesis submitted in conformity with the requirements for the degree of Master of Science Graduate Department University of Toronto. https://tspace.library.utoronto.ca/bitstream/1807/98105/3/Laliberte_Catherine_\%20_201911_MSc_thesis.pdf

- $\quad$ Lin W, Chen I, Wei F, et al. Clinical Significance of Preoperative Squamous Cell Carcinoma Antigen in Oral-Cavity Squamous Cell Carcinoma. Laryngoscope, 2011, 121:971-977.

- Navazesh M. Methods for collecting saliva. Jam N.Y.Acad Sci. 1993. 20: 72-77.

- Omura K, "Current status of oral cancer treatment strategies: surgical treatments for oral squamous cell carcinoma," International Journal of Clinical Oncology, vol. 19, no. 3, pp. 423-430, 2014.

- $\quad$ Pellicioli A.C.A., Bingle L., Farthing P., Lopes M. A., Martins M. D., and Vargas P. A., "Immunosurveillance profile of oral squamous cell carcinoma and oral epithelial dysplasia through dendritic and T-cell analysis," Journal of Oral Pathology \& Medicine, vol. 46, no. 10,pp. 928-933, 2017.

- Radhika T, Jeddy N, Nithya S, Muthumeenakshi RM. Salivary biomarkers in oral squamous cell carcinoma - An insight. J Oral Biol Craniofac Res. 2016;6(Suppl 1): S51S54. doi: 10.1016/j.jobcr.2016.07.003

- $\quad$ Rivera C, Venegas B. Histological and molecular aspects of oral squamous cell carcinoma (Review). Oncol Lett. 2014;8(1):7-11. doi:10.3892/ol.2014.2103.

- $\quad$ Sun A and Chiang C. Levamisole and/or Chinese medicinal herbs can modulate the serum level of squamous cell carcinoma associated antigen in patients with erosive oral lichen planus. J Oral Pathol Med 2001: 30: 542-548.

- $\quad$ Tampa M, Mitran M, Mitran CI, Sarbu MI, Matei C, Nicolae I, et al. Mediators of Inflammation - A Potential Source of Biomarkers in Oral Squamous Cell Carcinoma Journal of Immunology Research. Vol 2018, Article ID 1061780 , 12 pages. Tampa et al., 2018

- Watanabe Y, Yamaguchi Y, Komitsu N, S. Ohta S, Azuma, Izuhara Yand Aihara M Elevation of serum squamous cell carcinoma antigen 2 in patients with psoriasis: associations with disease severity and response to the treatment. British Journal of Dermatology 2016, 174, pp1327-1336. 